\title{
Relações de variáveis ambientais e subperíodos na produtividade e teor de óleo em canola
}

\author{
Relations of environments variables and subperiods in yield and content oil in canola
}

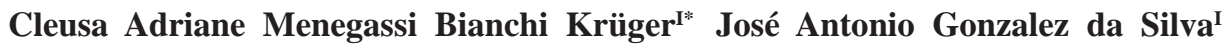 \\ Sandro Luis Petter Medeiros ${ }^{\mathrm{II}}$ Genei Antonio Dalmago ${ }^{\mathrm{III}}$ Adair José da Silva \\ Emílio Ghisleni Arenhardt ${ }^{\mathrm{I}}$ Ewerton Gewehr $^{\mathrm{I}}$
}

RESUMO

O objetivo do estudo foi avaliar as relações existentes entre a produtividade de grãos, teor de óleo e subperíodos da canola com a soma térmica (ST, ${ }^{\circ} \mathrm{C}$ dias) e a insolação real acumulada (IAC, horas) durante a fase de enchimento de grãos, além de classificar a importância dessas variáveis sobre as diferenças morfológicas observadas. Foram realizados três experimentos, com espaçamentos entre linhas de 0.20, 0.40 e 0.60m. Durante dois anos de cultivo (2008 e 2009), os genótipos 'Hyola 432' (precoce) e 'Hyola 61'(médio) foram avaliados em três densidades de plantio (20, 40 e 60 plantas $\mathrm{m}^{-2}$ ), em cada experimento. $O$ delineamento experimental utilizado foi o de blocos ao acaso, em arranjo fatorial $2 \times 2 \times 3$ (anos $x$ genótipos $x$ densidades), com quatro repetições. A soma térmica no subperíodo da floração à maturação fisiológica não está diretamente associada ao rendimento e teor de óleo nos grãos, frente ao arranjo de plantas. Os dias da emergência ao início da floração, da emergência à floração final e o tempo de duração da floração em canola são as variáveis que mais contribuem para a variação morfológica total.

Palavras-chave: Brassica napus, fases fenológicas, soma térmica, insolação, espaçamento entre linhas.

\section{ABSTRACT}

The objective of the study was to evaluate the relationship among grain yield, oil content and subperiods of canola with thermal time and accumulated sunshine hours during of grain-filling phase and classify the importance of this variables about morphological differences observed. Three experiments were carried out with row spacing of 0.20, 0.40 and $0.60 \mathrm{~m}$. During two crop years (2008 and 2009), the genotypes 'Hyola 432' and 'Hyola 61 'were evaluated in three plant densities (20, 40 e 60 plants $\left.\mathrm{m}^{-2}\right)$ in each experiment. The experimental design was to randomized blocks in factorial arrangement $2 \times 2 \times 3$ (years $x$ genotypes $x$ densities), with four replicates. The thermal time in subperiod of flowering to physiological maturation is not associated directly to the yield and oil content in grains by plant arrangement. The days of emergence to the beginning of flowering, of emergence to late flowering and duration time of flowering in canola are variables more contribute to morphological variation total.

Key words: Brassica napus, phenological phases, thermal sum, sunshine hours, row spacing.

\section{INTRODUÇÃO}

A canola é uma espécie de produção de grãos e de óleo de excelente qualidade durante a estação fria do ano na região Sul do Brasil (TOMM, 2007). No entanto, apresenta amplo período de floração e consequente maturação desuniforme (MARCHIORI JR et al., 2002). O arranjo de plantas pode ser uma alternativa para se alcançar aumento da uniformidade de plantas, produção de grãos e óleo nesta espécie (MOMOH \& ZHOU, 2001). Em canola de primavera, cultivada no sul do Brasil, as variações na duração do ciclo são determinadas pela temperatura do ar, mostrando que a soma térmica é a variável que melhor determina a alteração da duração dos subperíodos de desenvolvimento (DALMAGO et al., 2009). Embora a temperatura do ar e o fotoperíodo sejam os principais fatores que atuam sobre o desenvolvimento vegetal, a radiação solar também é decisiva nos processos fotomorfogenéticos e fotossintéticos (KUNZ et al.,

IDepartamento de Estudos Agrários (DEAg), Universidade Regional do Noroeste do Estado do Rio Grande do Sul (UNIJUÍ), 98700-000, Ijuí, RS, Brasil. E-mail: cleusa_bianchi@yahoo.com.br. *Autor para correspondência.

IIDepartamento de Fitotecnia, Universidade Federal de Santa Maria (UFSM), Santa Maria, RS, Brasil.

IIIEmpresa Brasileira de Pesquisa Agropecuária (EMBRAPA TRIGO), Passo Fundo, RS, Brasil. Recebido 20.12.12 Aprovado 17.02.14 Devolvido pelo autor 22.06.14 CR-2012-1331.R2 
2007). A disponibilidade de radiação solar influencia no conteúdo e qualidade do óleo, associada também ao efeito direto da temperatura do ar, conforme citam LANNA et al. (2005), para a soja. A radiação solar continua sendo uma variável não disponível em várias regiões do Brasil, diferentemente da insolação real, que é o dado mais facilmente obtido e associado à disponibilidade de radiação solar, conforme demonstrado por DORNELAS et al. (2006) pela equação de Angström-Prescott.

Medidas de correlação entre caracteres da planta com a produtividade de grãos têm sido empregadas como forma de auxiliar no entendimento destas relações como subsídio no aumento de produtividade das culturas. Portanto, possibilitam o conhecimento dos reflexos da alteração de um caráter sobre a expressão de outro com ele relacionado (BENIN et al., 2009). Além disso, pelo modelo de Singh (CRUZ \& CARNEIRO, 2003), é possível conhecer a importância relativa de caracteres, classificando as variáveis estudadas de acordo com a sua contribuição para a diferença morfológica total.

A modificação do arranjo de plantas e o entendimento dos elementos meteorológicos na expressão dos subperíodos, produtividade e teor de óleo em canola são informações importantes na melhoria das técnicas de manejo sobre esta espécie. O objetivo do estudo foi avaliar as relações existentes entre a produtividade de grãos, teor de óleo e subperíodos da canola com a soma térmica e a insolação real acumulada durante a fase de enchimento de grãos e classificar a importância destas variáveis sobre as diferenças morfológicas observadas.

\section{MATERIAL E MÉTODOS}

Os experimentos foram realizados nos anos de 2008 e 2009, no Instituto Regional de Desenvolvimento Rural (IRDeR), do Departamento de Estudos Agrários (DEAg) da Universidade Regional do Noroeste do Estado do Rio Grande do Sul (UNIJUÍ), em Augusto Pestana, RS (28²6'S, $54^{\circ} 00^{\prime} \mathrm{W}$; altitude de $298 \mathrm{~m}$ ). O solo da área experimental é um Latossolo Vermelho distroférrico típico. O clima da região, segundo classificação de Köppen, é do tipo Cfa (subtropical). O trabalho envolveu a realização de três experimentos, alterando em cada um deles o espaçamento entre linhas $(0.20$, 0.40 e $0.60 \mathrm{~m})$. Em cada experimento, empregouse o delineamento de blocos ao acaso, com quatro repetições, em arranjo fatorial $2 \times 2 \times 3$, sendo os fatores ano de cultivo (2008 e 2009), genótipo ('Hyola 432'-precoce e 'Hyola 61'-médio) e densidade de plantas $\left(20,40\right.$ e 60 plantas $\left.\mathrm{m}^{-2}\right)$. As parcelas eram de cinco linhas de cinco metros de comprimento, com área de 5,10 e $15 \mathrm{~m}^{2}$, nos espaçamentos de 0.20, 0.40 e $0.60 \mathrm{~m}$, respectivamente. A semeadura foi realizada de forma manual, de forma a obter as densidades de plantas desejadas.

Foram quantificadas a produtividade de grãos (PG, $\mathrm{kg} \mathrm{ha}^{-1}$ ) e o teor de óleo no grão (TO, \%) (PAQUOT, 1979), além das observações fenológicas: dias da emergência ao início da floração (DEIF), dias da emergência ao final da floração (DEFF), quando não havia mais flores abertas; tempo de duração de floração (TDF), obtido pela diferença entre o DEIF e DEFF; dias da emergência à maturação (DEM) quando 40 a $60 \%$ dos grãos da planta apresentaram troca da cor verde para marrom; dias do início da floração à maturação (DIFM) e dias do final da floração à maturação (DFFM).

Os dados meteorológicos foram obtidos na estação meteorológica convencional, instalada a $500 \mathrm{~m}$ da área experimental. Foi realizado o cálculo da soma térmica diária (STd) e da soma térmica acumulada (ST) pelas equações: STd= $\mathrm{Tm}-\mathrm{Tb}$ e $\mathrm{ST}=\sum \mathrm{STd}$, conforme ARNOLD, (1960), em que Tm é a temperatura média diária do ar e $\mathrm{Tb}$ é a temperatura basal para a canola $\left(\mathrm{Tb}=5^{\circ} \mathrm{C}\right)$, de acordo com DALMAGO et al. (2009). Tanto a soma térmica ( $\mathrm{ST} \mathrm{em}{ }^{\circ} \mathrm{C}$ dia) quanto a insolação acumulada (IAC, em horas) foram computadas do final da floração à maturação fisiológica. As análises realizadas envolveram de forma conjunta a variação de cultivares de canola (efeito genético) e dos anos de cultivo e densidade de plantas (efeito ambiental) na elaboração dos modelos estatísticos para cada experimento. Os dados foram submetidos à correlação de Pearson para obtenção da magnitude e sentido de associações entre as variáveis estudadas (CRUZ \& CARNEIRO, 2003). A significância das correlações foi avaliada a $5 \%$ de probabilidade de erro. Com base na análise multivariada pela distância generalizada de Mahalanobis $\left(\mathrm{D}^{2}\right)$, foi estimada a magnitude de contribuição relativa das variáveis, segundo critério de Singh (CRUZ \& CARNEIRO, 2003).

\section{RESULTADOS E DISCUSSÃO}

No espaçamento de $0.20 \mathrm{~m}$, associações entre DEFFe TDF com a PG foram negativas (Tabela 1). Por outro lado, o aumento do DEIF e do DFFM proporcionou efeito positivo sobre a PG. Nos demais espaçamentos (0.40 e $0.60 \mathrm{~m})$, foram observadas relações diretas e positivas do DFFM com a PG, portanto, o aumento da duração desse subperíodo é uma estratégia importante para o 
Tabela 1 - Coeficiente de correlação de Pearson entre variáveis ambientais, subperíodos e de produção de canola, cultivada em distintos espaçamentos entre linhas (0.20, 0.40 e 0.60m). Augusto Pestana, 2012.

\begin{tabular}{|c|c|c|c|c|c|c|c|c|c|}
\hline Variável & TO & DEIF & DEFF & TDF & DEM & DIFM & DFFM & ST & IAC \\
\hline PG & $0,42 *$ & $0,53 *$ & $-0,48 *$ & $-0,81 *$ & $-0,21$ & 0,19 & $0.60 *$ & $-0,25$ & 0,35 \\
\hline TO & - & $-0,17$ & $-0,58^{*}$ & $-0,26$ & $-0.60 *$ & $-0.40 *$ & $-0,04$ & $-0,61^{*}$ & 0,17 \\
\hline DEIF & - & - & 0,21 & $-0,72 *$ & $0,58 *$ & $0,80 *$ & $0,81^{*}$ & $0,47^{*}$ & $0,67 *$ \\
\hline DEFF & - & - & - & $0,51 *$ & $0,89 *$ & $0,58^{*}$ & $-0,23$ & $0,70^{*}$ & 0,27 \\
\hline TDF & - & - & - & - & 0,11 & $-0,29^{*}$ & $-0,87^{*}$ & 0,08 & $-0,39 *$ \\
\hline DEM & - & - & - & - & - & $0,80 *$ & 0,22 & $0,86^{*}$ & $0,52 *$ \\
\hline DIFM & - & - & - & - & - & - & $0,49 *$ & $0,56^{*}$ & $0,85^{*}$ \\
\hline DFFM & - & - & - & - & - & - & - & 0,34 & $0,54 *$ \\
\hline ST & - & - & - & - & - & - & - & - & $0,37^{*}$ \\
\hline PG & $-0,08$ & 0,19 & $-0,55^{*}$ & $-0,57^{*}$ & $-0,33^{*}$ & 0,05 & $0,45^{*}$ & -0.20 & $-0,08$ \\
\hline TO & - & $-0,46^{*}$ & $-0,11$ & $0,33^{*}$ & $-0,29^{*}$ & $-0,56^{*}$ & $-0,47^{*}$ & $-0,19$ & $-0,45^{*}$ \\
\hline DEIF & - & - & 0,25 & $-0,71^{*}$ & $0,56^{*}$ & $0,80^{*}$ & $0,86^{*}$ & $0,41^{*}$ & $0,64^{*}$ \\
\hline DEFF & - & - & - & $0,49 *$ & $0,85^{*}$ & $0,44^{*}$ & $-0,08$ & $0,61^{*}$ & $0,33 *$ \\
\hline TDF & - & - & - & - & 0,10 & $-0.40^{*}$ & $-0,83^{*}$ & 0,07 & $-0,33^{*}$ \\
\hline DEM & - & - & - & - & - & $0,69 *$ & $0,35^{*}$ & $0,89^{*}$ & $0,62 *$ \\
\hline DIFM & - & - & - & - & - & - & $0,68^{*}$ & $0,48^{*}$ & $0,82 *$ \\
\hline DFFM & - & - & - & - & - & - & - & $0,32 *$ & $0,47 *$ \\
\hline ST & - & - & - & - & - & - & - & - & $0,52 *$ \\
\hline PG & 0,11 & 0,10 & $-0,57^{*}$ & $-0,51^{*}$ & $-0,38^{*}$ & $-0,03$ & $0,54 *$ & $-0,13$ & 0,11 \\
\hline TO & - & $-0,53^{*}$ & $-0,19$ & 0,24 & $-0,23$ & $-0,68 *$ & $-0,26$ & 0,09 & $-0,51^{*}$ \\
\hline DEIF & - & - & 0,12 & $-0,64^{*}$ & $0,34 *$ & $0,83 *$ & $0,66^{*}$ & 0,06 & $0,55^{*}$ \\
\hline DEFF & - & - & - & $0,67^{*}$ & $0,85^{*}$ & $0,42 *$ & $-0,54^{*}$ & $0,47^{*}$ & 0,16 \\
\hline TDF & - & - & - & - & $0.40 *$ & $-0,29 *$ & $-0,91^{*}$ & $0,32 *$ & $-0,28^{*}$ \\
\hline DEM & - & - & - & - & - & $0,58 *$ & $-0,23$ & $0,79^{*}$ & $0,51 *$ \\
\hline DIFM & - & - & - & - & - & - & $0,36^{*}$ & 0,18 & $0.60 *$ \\
\hline DFFM & - & - & - & - & - & - & - & $-0,14$ & $0,38 *$ \\
\hline ST & - & - & - & - & - & - & - & - & $0,49 *$ \\
\hline
\end{tabular}

* Significativo a $5 \%$ de probabilidade de erro. $\mathrm{r}_{\mathrm{F}}=$ coeficiente de correlação de Pearson; $\mathrm{PG}=$ Produtividade de grãos, em kg ha ${ }^{-1}$; $\mathrm{TO}=$ teor de óleo, em \%; DEIF= dias da emergência ao início da floração; DEFF= dias da emergência ao final da floração; TDF= tempo de duração da floração; DEM= dias da emergência à maturação; DIFM= dias do início da floração à maturação; DFFM= dias da floração final à maturação; $\mathrm{ST}=$ soma térmica dos dias da floração final à maturação, em ${ }^{\circ} \mathrm{C}$ dias e $\mathrm{IAC}=$ insolação acumulada no período da floração final à maturação, em horas.

aumento da PG em canola. As correlações entre a PG com o DEM foram negativas nos espaçamentos de 0.40 e $0.60 \mathrm{~m}$, sugerindo que o incremento do ciclo da canola determina redução da PG nos referidos espaçamentos.
Nesse sentido, corrobora a constatação de BENIN et al. (2009) de quê o aumento do ciclo de cultivo em trigo não incrementou a $\mathrm{PG}$, pois o aumento do ciclo geraria um gasto energético extra para manutenção da planta, 
restando menos fotoassimilados para o enchimento de grãos. Além disso, cultivares de canola de inverno mostraram relação negativa do atraso da semeadura com a duração do DFFM, fato que determinou menor PG (ROBERTSON et al., 2004). Porém, estudando variedades de polinização aberta de canola, COIMBRA et al. (2004) observaram relação direta e positiva entre o ciclo total e a PG.

No espaçamento de $0.20 \mathrm{~m}$ (Tabela 1 ), foi observada correlação positiva da PG com o TO, indicando que esse espaçamento entre linhas é o mais responsivo na relação positiva entre grãos e óleo. A PG não mostrou relação com as variáveis ST e IAC, presumindo-se que os níveis ocorridos de temperatura do ar e insolação real não influenciaram diretamente a PG.

O incremento dos subperíodos DEFF, DIFM e do DEM apresentaram correlação negativa com o TO no espaçamento de $0.20 \mathrm{~m}$ entre linhas (Tabela 1). Além disso, o TO mostrou relação inversa com a ST, indicando que anos com ST mais elevadas reduzem o TO. Em soja, LANNA et al. (2005) observaram menor TO quando a temperatura do ar foi mais elevada, corroborando a tendência observada neste estudo. Possivelmente, a elevação da temperatura do ar incrementa a respiração de manutenção, restando menos fotoassimilados para a formação dos lipídios. A relação entre TO e TDF não apresentou o mesmo padrão de resposta para os três espaçamentos, sendo a relação positiva significativa somente para o de $0.40 \mathrm{~m}$. Portanto, o maior TDF, assim como o ciclo total relatado como vantagem nesta espécie por diminuir os danos decorrentes de altas temperaturas e geada, podem não refletir em maior TO e PG, conforme observado nas relações negativas verificadas com estas variáveis.

Foi observada relação inversa e significativa entre o DEIF e o DIFM com TO em todos os espaçamentos (Tabela 1), exceto para DEIF em 0,2m. Ressalta-se que a tendência de que a diminuição do ciclo favoreceria o aumento do TO nos grãos deve ser vista com cautela. A redução acentuada do ciclo acarreta menor área foliar, resultando menor produção de fotoassimilados para formação de lipídios. Por outro lado, o aumento do ciclo implica maior gasto de manutenção, restando menor quantidade de fotoassimilados para serem translocados durante o enchimento de grãos. Como o gasto energético para a formação do óleo é maior do que aquele necessário para a formação do restante dos grãos, tanto a redução acentuada como crescimento excessivo do ciclo podem ser prejudiciais para TO.

Ocorreu relação inversa e significativa de TO com o IAC nos espaçamentos de 0.40 e $0.60 \mathrm{~m}$
(Tabela 1). Essa resposta está relacionada ao fato de que o aumento do número de horas de radiação solar determina elevação da temperatura do ar, devido à maior disponibilidade de energia. Assim, a partir do ponto de saturação luminosa, os acréscimos de radiação elevam a temperatura do ar, acarretando diminuição da fotossíntese líquida devido ao aumento da taxa de respiração, criando uma condição desfavorável ao acúmulo de óleo nos grãos. A relação entre horas sol e temperatura do ar encontra-se fortalecida pela relação direta do IAC com a ST em todos os espaçamentos estudados, em vista de que a maior insolação determinou, consequentemente, maior elevação da temperatura do ar. $\mathrm{O}$ efeito direto da temperatura do ar sobre a produção de óleo também foi verifica por HASSAN et al. (2005), os quais comentam que a fase reprodutiva longa associada à baixa temperatura do ar durante o enchimento de grãos foram decisivas para a maior PG e qualidade de óleo em canola de inverno.

Em geral, as associações existentes entre as fases fenológicas da canola e ST foram positivas nos três espaçamentos, exceto para o TDF que somente foi significativa em $0.60 \mathrm{~m}$ (Tabela 1). Apesar de ST ter evidenciado maior efetividade sobre o TDF no espaçamento de $0.60 \mathrm{~m}$, existe tendência do aumento da TDF em comprometer a PG, como observado, na relação PG x TDF. Esse fato foi constatado por CABRAL et al. (2011) na análise de trilha em feijão, na qual verificaram que o período de florescimento foi a variável secundária de influência negativa sobre a PG. Acrescenta-se ainda que NAVARRO JÚNIOR \& COSTA (2002) confirmaram que cultivares de soja com período de floração longo não apresentaram maior PG, pois, ao mesmo tempo em que ocorre a produção e fixação de legumes, ocorre a formação de outras estruturas da planta. Dessa forma, assim como ocorre em soja, a canola também evidencia a sobreposição de estádios fenológicos, influenciando a competição entre estruturas reprodutivas e contribuindo na expressão da PG.

A variável TDF, além de mostrar relação negativa com a PG nos três espaçamentos estudados, também apresentou relação significativa e inversa com a IAC. Portanto, a relação anteriormente relatada entre ST com a IAC parece fortalecer a hipótese da associação entre maior insolação e a elevação da temperatura do ar. Nesse sentido, DALMAGO et al. (2009) mencionam que a temperatura do ar, via ST, é o principal fator determinante da duração dos subperíodos da canola.

$\mathrm{Na}$ tabela 2, os valores de PG foram maiores nos espaçamentos de 0.20 e $0.40 \mathrm{~m}$ quando 
Tabela 2 - Estatística descritiva e contribuição relativa de variáveis ambientais, fenológicas e de produção sob diferentes espaçamentos entre linhas $(0.20,0.40$ e $0.60 \mathrm{~m})$. Augusto Pestana, 2012.

\begin{tabular}{|c|c|c|c|c|}
\hline \multirow{2}{*}{ Variável } & \multicolumn{2}{|c|}{ 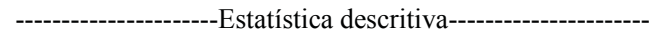 } & \multicolumn{2}{|c|}{----Contribuição relativa-- } \\
\hline & Valor médio & Desvio padrão & sj & sj $(\%)$ \\
\hline PG & 969,0 & 320,0 & 444,67 & 0,02 \\
\hline TO & 28,0 & 5,1 & $15.570,61$ & 0,36 \\
\hline DEIF & 65,0 & 7,6 & $1.804 .422,67$ & 42,85 \\
\hline DEFF & 111,0 & 6,1 & $1.875 .857,96$ & 44,55 \\
\hline TDF & 45,0 & 8,6 & $150.479,98$ & 3,57 \\
\hline DEM & 146,0 & 6,1 & $215.454,50$ & 5,11 \\
\hline DIFM & 88,0 & 4,5 & $5.243,14$ & 0,15 \\
\hline DFFM & 34,0 & 2,7 & $93.218,65$ & 2,21 \\
\hline ST & 445,0 & 38,5 & $15.778,34$ & 0,38 \\
\hline IAC & 213,0 & 30,3 & $33.714,71$ & 0,80 \\
\hline PG & 948,0 & 249,4 & $1.171,49$ & 0,02 \\
\hline TO & 26,0 & 4,6 & $2.790,30$ & 0,02 \\
\hline DEIF & 67,0 & 7,3 & 3.992.627.262,99 & 49,85 \\
\hline DEFF & 111,0 & 5,9 & 2.567.224.776,29 & 32,14 \\
\hline TDF & 44,0 & 8,2 & 1.425.293.092,93 & 17,84 \\
\hline DEM & 146,0 & 6,7 & $264.140,47$ & 0,02 \\
\hline DIFM & 88,0 & 5,4 & $26.192,10$ & 0,02 \\
\hline DFFM & 34,0 & 2,8 & $14.376,27$ & 0,03 \\
\hline ST & 454,0 & 56,3 & $13.852,79$ & 0,03 \\
\hline IAC & 219,0 & 34,4 & $28.305,44$ & 0,03 \\
\hline PG & 834,0 & 171,1 & 477,10 & 0,03 \\
\hline TO & 27,0 & 4,7 & 14,45 & 0,03 \\
\hline DEIF & 67,0 & 5,9 & $16.623 .203 .345,34$ & 49,84 \\
\hline DEFF & 111,0 & 6,1 & $442.527 .263,90$ & 13,30 \\
\hline TDF & 43,0 & 7,9 & $1.219 .740 .511,87$ & 36,68 \\
\hline DEM & 148,0 & 5,7 & $383.924,27$ & 0,03 \\
\hline DIFM & 87,0 & 5,0 & $3.748,05$ & 0,03 \\
\hline DFFM & 35,0 & 1,8 & 18,91 & 0,02 \\
\hline ST & 477,0 & 46,9 & $51.588,24$ & 0,02 \\
\hline IAC & 227,0 & 33,3 & $19.073,46$ & 0,02 \\
\hline
\end{tabular}

s. $\mathrm{j}$.= contribuição relativa; $\mathrm{PG}=$ Produtividade de grãos, em $\mathrm{kg} \mathrm{ha}^{-1} ; \mathrm{TO}=$ teor de óleo, em \%; DEIF= dias da emergência ao início da floração; $\mathrm{DEFF}=$ dias da emergência ao final da floração; TDF= tempo de duração da floração, em dias; DEM= dias da emergência à maturação; DIFM= dias do início da floração à maturação; DFFM= dias da floração final à maturação; ST = soma térmica dos dias da floração final à maturação, em ${ }^{\circ} \mathrm{C}$ dias e $\mathrm{IAC}=$ insolação acumulada no período da floração final à maturação, em horas.

comparado ao de $0.60 \mathrm{~m}$. RAMBO et al. (2003) destacam que, em espaçamentos entre linhas maiores, a soja tende a apresentar menor PG. THOMAS (2003), em canola de inverno, observou maior PG no menor espaçamento entre linhas, também observado neste estudo. Os TO nos três espaçamentos mostraram um comportamento similar, indicando uma estabilidade no caráter com média ao redor $27 \%$. Esses valores estão abaixo daqueles referenciados na literatura para a espécie com valores ao redor de $38 \%$ de óleo (ROBERTSON et al., 2004; HASSAN et al., 2005). Em canola, LEACH et al. (1999) não constataram alteração no TO em distintas densidades de plantas, porém, atribuíram ao espaçamento entre linhas a 
maior alteração no caráter. Na análise da contribuição relativa frente à variabilidade morfológica total (s.j.; $\%$ os espaçamentos de 0.20 e $0.40 \mathrm{~m}$ mostraram maior contribuição dos DEIF e DEFF (Tabela 2). Por outro lado, o TDF foi incrementado de $3,57 \%$ no espaçamento $0.20 \mathrm{~m}$ para $17,84 \%$, no de $0.40 \mathrm{~m}$. Constata-se que o aumento do espaçamento entre linhas promoveu alterações que se compensam nestas duas variáveis de forma conjunta a alterar resposta da planta para ajuste no dossel. A canola é uma espécie de hábito de crescimento indeterminado e que apresenta estruturas plásticas e ajustáveis na população (KRÜGER et al., 2011), assim como verificado também por JACOB JÚNIOR et al. (2012).

A fase vegetativa (DEIF) mostrou elevada contribuição e estabilidade, com similaridade de valores nos três espaçamentos $(0.20,0.40$ e $0.60 \mathrm{~m}$ ) (Tabela 2). Por outro lado, houve redução da contribuição relativa sobre o caráter DEFF e o direcionamento no incremento no TDF, na condição de maior espaçamento $0.60 \mathrm{~m}$. Contudo, como observado na tabela 1 , o incremento do TDF promoveu efeitos negativos sobre a PG, de tal forma que o aumento do TDF reduz a PG. Ressalta-se neste estudo que a TDF representa caráter alvo de observação para melhoristas e ecofisiologistas desta espécie por sua alteração frente às condições de ambiente e ligação direta negativa com a PG.

\section{CONCLUSÃO}

A soma térmica no subperíodo da floração à maturação fisiológica não está diretamente associada ao rendimento e teor de óleo nos grãos frente ao arranjo de plantas.

Os dias da emergência ao início da floração, da emergência à floração final e o tempo de duração da floração em canola são as variáveis que mais contribuem para a variação morfológica total.

\section{AGRADECIMENTO}

Ao Conselho Nacional de Desenvolvimento Científico e Tecnológico $(\mathrm{CNPq})$ e à UNIJUÍ, pelo aporte de recursos destinados a este estudo e pelas bolsas de iniciação científica e produtividade em pesquisa.

\section{REFERÊNCIAS}

ARNOLD, C.Y. Maximum-minimum temperatures as a basis for computing heat units. Proceedings of the American Society for Horticultural Sciences, v.76, p.682-692, 1960. Disponível em: $<$ http://www.cabdirect.org/abstracts/19610305608.html;jsessionid $=1 \mathrm{CBFD} 9 \mathrm{FFB} 5 \mathrm{~B} 2 \mathrm{D} 27481 \mathrm{AE} 2605 \mathrm{BDE} 48 \mathrm{~A} 6 \mathrm{~F}>$. Acesso em: 25 ago. 2011.
BENIN, G. et al. Capacidade de combinação em genótipos de trigo estimada por meio de análise multivariada. Pesquisa Agropecuária Brasileira, v.44, n. 9, p.1145-1151, 2009. Disponível em: <http:// www.scielo.br/pdf/pab/v44n9/v44n9a12.pdf>. Acesso em: 15 nov. 2011. doi: 10.1590/S0100-204X2009000900012.

CABRAL, P.D.S. et al. Análise de trilha do rendimento de grãos de feijoeiro (Phaseolus vulgaris L.) e seus componentes. Revista Ciência Agronômica, v.42, n. 1, p. 132-138, 2011. Disponível em: $<$ http://www.scielo.br/pdf/rca/v42n1/v42n1a17.pdf $>$. Acesso em: 22 nov. de 2011. doi: 10.1590/S1806-66902011000100017.

COIMBRA, J.L.M. et al. Análise de trilha dos componentes do rendimento de grãos em genótipos de canola. Ciência Rural, v.34, n. 5, p.1421-1428, 2004. Disponível em: <http://www.scielo. br/pdf/cr/v34n5/a15v34n5.pdf>. Acesso em: 28 set. 2011. doi: 10.1590/S0103-84782004000500015.

CRUZ, C.D.; CARNEIRO, P.C.S. Modelos biométricos aplicados ao melhoramento genéticos. Viçosa, MG: UFV, 2003. 585p.

DALMAGO, G.A. et al. Canola. In: MONTEIRO, J.EB.A. (Org.). Agrometeorologia dos cultivos. Brasília: INMET, 2009. V.1, p.131-149.

DORNELAS, K.D. e S. et al. Coeficientes médios da equação de Angström-Prescott, radiação solar e evapotranspiração de referência em Brasília. Pesquisa Agropecuária Brasileira, v.41, n.8, p.1213-1219, 2006. Disponível em: <http://www.scielo. br/pdf/pab/v41n8/31695.pdf>. Acesso em: 25 out. 2011. doi: 10.1590/S0100-204X2006000800001.

HASSAN, F.U. et al. Effects of environmental variation on oil content and fatty acid composition of canola cultivars. Journal of Research (Science), v.16, n. 2, p.66-72, 2005. Disponível em: <http://bzu.edu. pk/jrscience/vol16no2/9.pdf> Acesso em: 20 de out. 2011.

KRÜGER, C.A.M.B. et al. Herdabilidade e correlação fenotípica de caracteres relacionados à produtividade de grãos e à morfologia da canola. Pesquisa Agropecuária Brasileira, v.46, n. 12, p.1625-1632, 2011. Disponível em: <http://www.scielo.br/pdf/ pab/v46n12/46n12a07.pdf >. Acesso em: 20 mar. 2012. doi: 10.1590/S0100-204X2011001200007.

KUNZ, J.H. et al. Uso da radiação solar pelo milho sob diferentes preparos do solo, espaçamento e disponibilidade hídrica. Pesquisa Agropecuária Brasileira, v.42, n. 11, p.15111520, 2007. Disponível em: <http://www.scielo.br/pdf/pab/ v41n8/31695.pdf>. Acesso em: 25 maio 2012. doi: 10.1590/ S0100-204X2007001100001.

JACOB JÚNIOR, E.A. et al. Changes in canola plant architecture and seed physiological quality in response to different sowing densities. Revista Brasileira de Sementes, v.34, n. 1, p.14-20, 2012. Disponível em: <http://www.scielo.br/pdf/rbs/v34n1/a02v34n1.pdf>. Acesso em: 20 ago. 2012. doi: 10.1590/S0101-31222012000100002.

LANNA, A.C. et al. Effect of temperature on polyunsaturated fatty acid accumulation in soybean seeds. Brazilian Journal of Plant Physiology, v.17, n. 2, p.213-222, 2005. Disponível em: <http:// www.scielo.br/pdf/bjpp/v17n2/a04v17n2.pdf>. Acesso em: 20 ago. 2011. doi: 10.1590/S1677-04202005000200004.

LEACH, J.E. et al. Effects of high plant populations on the growth and yield of winter oilseed rape (Brassica napus). Journal of Agricultural Science, v.132, n.2, p. 173-180, 1999. Disponível 
em: <http://journals.cambridge.org/action/displayAbstract?fromP age $=$ online\&aid $=7251>$. Acesso em: 20 ago. 2011 .

MARCHIORI JR., O. et al. Qualidade e produtividade de sementes de canola (Brassica napus) após aplicação de dessecante em précolheita. Planta Daninha, v.20, n. 2, p.253-261, 2002. Disponível em: <http://www.scielo.br/pdf/pd/v20n2/12.pdf>. Acesso em: 20 out. 2011. doi: 10.1590/S0100-83582002000200012.

MOMOH, E.J.J.; ZHOU, W. Growth and yield responses to plant density and stage of transplanting in winter oilseed rape (Brassica napus L.). Journal Agronomy \& Crop Science, v.186, p.253259, 2001. Disponível em: <http://onlinelibrary.wiley.com/ doi/10.1046/j.1439-037x.2001.00476.x/pdf>. Acesso em: 15 set. 2011. doi: 10.1046/j.1439-037x.2001.00476.x.

NAVARRO JÚNIOR, H.M.: COSTA, J.A. Contribuição relativa dos componentes do rendimento para produção de grãos em soja. Pesquisa Agropecuária Brasileira, v.37, n. 3, p.269274, 2002. Disponível em: <http://www.scielo.br/pdf/pab/ v37n3/9000.pdf>. Acesso em: 20 out. 2011. doi: 10.1590/S0100204X2002000300007.
PAQUOT, C. Standard methods for the analysis of oils, fats and derivatives. 6.ed. Oxford: Pergamon, 1979. 170p.

RAMBO, L. et al. Rendimento de grãos da soja em função do arranjo de plantas. Ciência Rural, v.33, n. 3, p.405-411, 2003. Disponível em: <http://www.scielo.br/pdf/cr/v33n3/ a03v33n3.pdf>. Acesso em: 20 out. 2011. doi: 10.1590/S010384782003000300003

ROBERTSON, M.J. et al. Response of canola and Indian mustard to sowing date in the grain belt of North. Disponível em: <http://www.publish.csiro.au/paper/EA02214.htm>. Acesso em: 20 out. 2011. doi: 10.1071/EA02214.

THOMAS, P. Canola grower's manual. Winnipeg: Canola Council of Canada, 2003. Disponível: $<$ http://www.canolacouncil. org/canola_growers_manual.aspx>. Acesso em: 25 set. 2011.

TOMM, G.O. Indicativos tecnológicos para produção de canola no Rio Grande do Sul. Passo Fundo: Embrapa Trigo. 2007. 32p. Disponível em: <http://www.cnpt.embrapa.br/culturas/canola/p_ sp03 2007.pdf>. Acesso em: 10 maio 2011. 